Research Article

\title{
Economic Development Trend Prediction Model Based on Unsupervised Learning in the Internet of Things Environment
}

\author{
Min Kuang (iD \\ Business School, XinYang Vocational and Technical College, XinYang 464000, China \\ Correspondence should be addressed to Min Kuang; kuangmin66@xyvtc.edu.cn
}

Received 20 October 2021; Revised 6 November 2021; Accepted 23 November 2021; Published 8 December 2021

Academic Editor: Qiangyi Li

Copyright $\odot 2021$ Min Kuang. This is an open access article distributed under the Creative Commons Attribution License, which permits unrestricted use, distribution, and reproduction in any medium, provided the original work is properly cited.

\begin{abstract}
In order to explore the economic development trend under the environment of the Internet of Things, this paper improves the chaotic algorithm of the Internet of Things and constructs an economic development trend analysis system based on big data technology. Moreover, this paper analyzes the actual situation of big data processing data and conducts research on economic data analysis process. In addition, this paper conducts effective research on the various modules of functional analysis, obtains the system functional architecture, constructs the system functional structure based on the actual situation, and analyzes the operating process of the system. Finally, this paper designs a simulation test based on actual data. The experimental research results show that the system model proposed in this paper has a good performance in the forecast of economic development trends, and the system can be used for forecasting in subsequent economic development forecasts.
\end{abstract}

\section{Introduction}

With the continuous development of the world economy, our country's economy has entered a new era. The economic growth rate has gradually shifted from the original highspeed growth to the current medium-high growth. The new era corresponds to new development methods, and development gradually shifts its focus to optimize the industrial structure, transform the main body of the three major industries, accelerate the development of the tertiary industry, increase the scale of urbanization, improve people's living standards, and reduce the gap between urban and rural areas. At the same time, it is necessary to change the driving mode of economic development, strengthen innovation driving, and weaken factor driving and investment driving. Based on a comprehensive analysis of the world's past economic development and the characteristics of the world's economic development cycle, as well as the status quo of China's economic development, we recognize that the conversion of new and old kinetic energy is a process that our country's economic development must go through, and we must gradually adapt and seize the initiative to actively lead the conversion of new and old kinetic energy. At present, the Chinese economy cannot balance supply and demand well in the development process. The poor quality of products and services provided by the supply side has led to serious shortage on the demand side. The structural contradictions between the supply side and the demand side have become a stumbling block to the healthy and sustainable development of our country's economy. Therefore, our country must not only strengthen the quality of supplyside products and services, but also increase investment, consumption, and export demand, upgrade, and transform traditional industries, and release new space for kinetic energy. After experiencing the great economic crisis, developed countries have gradually realized that they need to return to the manufacturing industry to prevent the country from hollowing out the industry due to excessive outsourcing. The focus of the return is on industrial upgrading and the use of high-tech for high-end manufacturing. Therefore, the manufacturing industry in China's emerging markets is facing a major crisis.

In recent years, the main theme of economic development has been the upgrading of industrial structure. However, the upgrading of the industrial structure requires a certain amount of support from the external environment, especially 
the financial capital structure. Throughout the course of economic development, financial support has played a significant role in the process of irrational industrial structure to rationalization and industrial structure from low-level to high-level. The unbalanced distribution of financial capital is not uncommon in the process of financial marketization reform, and the degree of optimization of the financial structure is closely linked to the development trend of the national economy. First, the financial structure has been continuously optimized with economic development, and the optimization of the financial structure will in turn promote economic development. Secondly, the optimization of the financial structure is an effective way to promote macroeconomic growth, which is mainly reflected in two aspects: (1) By improving the capital allocation effect of the social production sector, it can achieve the promotion of technological progress and the optimization and upgrading of the industrial structure. (2) The optimization and upgrading of the industrial structure can promote social economic growth by increasing social productivity and increasing total social investment, thereby changing the financial structure and total financial volume. Optimizing the financial structure to promote economic development has always been a hot spot in financial theory and empirical analysis.

The relationship between finance and economic development is one of the topics continuously discussed in academia. With the maturity of information technology and the deepening of economic integration, various resources such as finance flow rapidly between regions and the phenomenon that financial institutions and financial activities are gradually concentrated in a particular region have appeared. From a global perspective, there are three wellknown financial clusters in the world: Shinjuku, Tokyo, City of London, and Wall Street in New York. From a domestic perspective, typical representatives of financial clusters include Beijing Financial Street, Shanghai Lujiazui, and Shenzhen Financial Center. In addition, some fund towns in other regions have unique characteristics. The agglomeration of regional industries gave birth to these financial agglomeration areas, forming a special industrial spatial organization model. In turn, financial agglomeration will also promote the development of the regional economy. For economic development itself, it should cover two aspects: the scale of development and the quality of development. Therefore, when judging the situation of social and economic development, we must comprehensively consider the scale and quality of economic development. Based on this, the question we want to study is the current status of my country's provincial financial agglomeration and comprehensive economic development and what kind of relationship exists between the two systems of financial agglomeration and economic development, so as to provide a scientific basis for promoting regional economic development.

Based on the above analysis, this paper analyzes the economic development trend in the Internet of Things environment, combines big data technology to carry out economic development data research, and predicts and analyzes economic development trends.

\section{Related Work}

In foreign countries, in view of the existence of different research angles and schools of thought on the issue of financial development supporting the upgrading of industrial structure, the conclusions are not unified. Literature [1] divided the economy as a whole into surplus, balance, and loss. If there are losses or surpluses between economic sectors, investors will inevitably carry out external financing to achieve a balance. In addition, external financing is divided into direct financing and indirect financing, namely, the issuance of direct securities in the financial market and the issuance of indirect securities through financial intermediaries. Literature [2] analyzed the basic process and laws of financial structure development and proposed that there is only one path for financial development: eliminating war and inflation. Literature [3] studied the relationship between financial structure and economic growth based on the theory of financial repression and deepening and pointed out that increasing the real income of money to increase the accumulation of money is conducive to the improvement of social economic monetization. Literature [4] showed that the bankingbased financial structure plays a more significant role in promoting economic growth. Literature [5] used the VAR model to conduct an empirical study on the relationship between financial institutions, stock markets, and economic development and carried out error corrections. It is found that there is a long-term balanced two-way causal relationship between banks and stock markets and economic development, and both can promote economic development. Although the degree of influence is small, the promotion of financial institutions is greater than that of the stock market. Literature [6] constructs a multipart enhancement model. Studies have shown that financial development is to support the development of emerging industries and promote the optimization of industrial structure by means of the flow of funds to units with advanced technology. Literature [7] concluded that financial development supports the upgrading of industrial structure to a certain level. Literature [8] found that financial deepening has a higher driving force for economic growth. Literature [9] found that the dominant potential of the market-led financial system lies in the aspects of information transparency, price function, promotion of technological progress, good risk management, and enterprise improvement management. Literature [10] believed that the institutional environment has an obvious regulatory role in the study of the financial-oriented effect and the role of the market-oriented financial system. Literature [11] pointed out that the close relationship between financial markets and banks should be fully utilized to guide financial structuring.

Literature [12] studied the impact of financial structure on the regional economy from three aspects: macro, meso, and micro. The microaspect is related to the scale of regional finance, the types of financial instruments, and the price composition of financial assets. The main distribution of institutions related to the formation of the financial market 
is the mesoscopic aspect. The macroaspect is related to the structure of the regional financial system and macropolicy objectives. Literature [13] analyzed the structure of China's financial assets from the currency level and summarized the reasons for the rapid rise of China's broad money and GDP. Literature [14] deeply discussed the impact of the number of financial systems and economic development on China, found the key to China's financial efficiency, and put forward a series of strategic recommendations. Literature [15] concluded through empirical research that the expansion of financial scale can promote economic development to a certain extent. In addition, given a certain scale of the financial system, the larger the scale of the nonbanking system, the greater the promotion of economic development. Literature [16] studied different financial structures that produce different economic growth performance based on information asymmetry. Literature [17] focused on the relationship between securities, assets, and economic growth. It is found that if the proportion of securities assets in the total securities assets (stocks and bonds) is higher, the economic growth rate will be faster. Literature [18] empirically showed that there is a long-term equilibrium relationship between financial structure and economic growth, and the Granger causality test finds that economic growth is the one-way Granger reason for the financial structure of Shandong Province. Finally, it is pointed out that the financial structure that promotes economic growth should be adjusted and optimized to provide the most suitable and effective financing method for the real economy. Literature [19] believed that there is an interaction mechanism between the structure of financial assets and the economic growth of a region or country. The two restrict each other and develop together. Literature [20] believed that the structure of different types of financial assets has different effects on economic growth. Among them, monetary assets and stocks play an important role in promoting economic growth, while bonds, insurance, and funds have no obvious impact on the economy. Literature [21] analyzed the factor endowment structure at different stages of economic development and concluded that, in the process of economic development, when the financial system structure and industrial structure are optimally matched, the financial structure plays the most obvious role in promoting economic development. Literature [22] used two aspects of direct finance and indirect finance to study the role of financial development in promoting the upgrading of industrial structure.

\section{Chaos Data Processing Algorithm of the Internet of Things}

At present, after in-depth research on the theory of data chaos in the Internet of Things, the chaotic dynamic system of data in the Internet of Things has received more and more attention from researchers. Up to now, the definition of data chaos in the Internet of Things is still divided. There are two generally accepted definitions: Li-Yorke IoT data chaos definition and Deveny IoT data chaos definition. Its brief introduction is as follows.
Definition 1. The definition of Li-Yorke IoT data chaos

Its setting $f$ is a continuous mapping from the closed interval I to itself. If the following conditions are met, it is said that $f$ is chaotic in the Li-Yorke IoT data in the closed interval I:

(1) There is no upper bound on the period of the $f$ period point.

(2) There is an uncountable subset $s$ on the closed interval I, which satisfies the following formula:

$$
\begin{aligned}
& \varlimsup_{n \longrightarrow \infty}\left|f^{n}(x)-f^{n}(y)\right|>0, \quad \forall x, y \in S, \\
& \lim _{n \longrightarrow \infty}\left|f^{n}(x)-f^{n}(y)\right|=0, \quad \forall x, y \in S, \\
& \varlimsup_{n \longrightarrow \infty}\left|f^{n}(x)-f^{n}(p)\right|>0, \quad \forall x \in S, p \in P(f),
\end{aligned}
$$

and among them, $\mathrm{x} \neq \mathrm{y}, \quad f^{0}(x)=x, f^{1}(x)=f$ $(x), \ldots, f^{n+1}(x)=f\left(f^{n}(x)\right), n \in N$, and the uncountable subset $S$ is a chaotic set of IoT data. The unsatisfied point $x$ in formula (2) is the asymptotic periodic point of $f$, and $P(f)$ represents the periodic point set of $f$.

Definition 2. Deveny IoT data chaos definition.

We set $X$ to be a metric space, and $\mathrm{f}: X \longrightarrow X$ is a continuous self-mapping on $\mathrm{X}$. If the following conditions are met, $f$ is said to be chaotic in Deveny IoT data on X:

(1) $f$ is transferable; that is, for any nonempty subsets $U$ and $\mathrm{V}$ of $X$, there is always a natural number $k$, which satisfies the following formula:

$$
f^{k}(U) \cap V \neq \varnothing .
$$

(2) The periodic point of $f$ is dense at $X$.

(3) $\mathrm{f}$ is highly sensitive to initial conditions; that is, if there is $\delta>0$ (sensitive constant), for any neighborhood $o$ of any $x \in X$ and $x$, there will always be $y \in O$ and a natural number $n$ satisfying the following formula:

$$
\left|f^{n}(x)-f^{n}(y)\right|>\delta .
$$

Although the definition of IoT data chaos has not yet been unified, compared with other nonlinear systems, IoT data chaos still exhibits its own unique characteristics, as follows.

3.1. Sensitivity to Initial Conditions. Lorenz used the term "butterfly effect" to properly describe the characteristics of the IoT data chaotic system that is sensitive to initial values. In the same IoT data chaotic system, two slightly different initial values are used for evolution. After a long period of evolution, the slight difference between the initial values has been continuously amplified, and the trajectories of the two will be completely irrelevant. 
3.2. The Overall Stability and Local Instability. Overall stability means that the performance of the system will not change due to small disturbances. However, even slight changes in the initial conditions can cause local instability.

3.3. Forking. When some control parameters of the system change, the new nonlinear solution will branch out, and the phase trajectory graph will also change. At this time, the system loses stability, and after multiple forks, it will eventually reach the chaotic state of IoT data.

3.4. Ergodicity. The ergodicity of IoT data chaos means that, within a certain time range, the trajectory of the IoT data chaotic movement traverses the position of each state point in the IoT data chaotic attractor without repeating it.

3.5. Boundedness. In the process of chaotic evolution of IoT data, although the chaotic trajectory of IoT data is chaotic and disorderly, the trajectory is always in a certain area and will not deviate from the scope of the chaotic attractor of IoT data and has certain regularity.

3.6. Inherent Randomness. In the data chaotic system of the Internet of Things, the random appearance of a certain motion state depends on its own internal reasons and is not subject to external disturbances.

3.7. Unpredictability. The unpredictable appearance of the chaotic system of IoT data is similar to random noise signals. This means that the motion state of the chaotic system of IoT data at a certain current moment is unpredictable and has no relation to the motion state of the previous moment and the next moment and does not have an obvious order state.

\subsubsection{One-Dimensional Logistic Mapping.}

One-dimensional logistic mapping was originally used to describe the law of population changes. Although the logistic mapping system is very simple, it has applications in many fields and is currently often used to analyze the laws of population changes. The system equation of one-dimensional logistic mapping is shown in the following formula:

$$
x_{n+1}=\mu x_{n}\left(1-x_{n}\right), \quad n=0,1,2 \ldots
$$

Among them, $x_{n} \in(0,1), \mu$ is the system control parameter, and $\mu \in(0,4]$. The bifurcation diagram is shown in Figure 1 . When $\mu \in(3.5699456,4]$, the system is in a state of data chaos in the Internet of Things. When $\mu$ is closer to 4 , the ergodicity and pseudo-randomness of each state are more obvious. Therefore, one-dimensional logistic mapping is very suitable for application in the field of information security.

3.7.2. Lorenz IoT Data Chaos System. When analyzing its dynamic behavior, it was found that there is a Lorenz attractor. The Lorenz system is shown in the following formula:

$$
\left\{\begin{array}{l}
\dot{x}_{1}=a\left(x_{2}-x_{1}\right), \\
\dot{x}_{2}=b x_{1}-x_{2}-x_{1} x_{3}, \\
\dot{x}_{3}=x_{1} x_{2}-c x_{3} .
\end{array}\right.
$$

Among them, $a, b$, and $c$ are system parameters. In the case of $a=10, b=28$, and $c=8 / 3$, the system enters the data chaos state of the Internet of Things. Figure 2 shows part of the phase diagram.

3.7.3. Chaos Lorenz System of Super IoT Data. The Lyapunov exponent can reflect the local instability of the chaotic attractor of IoT data. When the system has at least one positive Lyapunov exponent, the system is a chaotic system of IoT data. And some systems have more than one Lyapunov exponent greater than 0 , which is a hyper-IoT data chaotic system.

On the basis of the Lorenz IoT data chaotic system, the equations are further expanded to obtain a four-dimensional super IoT data chaotic Lorenz system. It is shown in the following formula:

$$
\left\{\begin{array}{l}
\dot{x}=a(y-x)+w, \\
\dot{y}=b x-y-x z, \\
\dot{z}=x y-c z, \\
\dot{w}=d w-y z .
\end{array}\right.
$$

Among them, a, $b, \mathrm{c}$, and $d$ are specific parameters. When $a=10, b=28, c=8 / 3$, and $d=-1$, there are two Lyapunov exponents that are greater than 0 , so the system is in a state of data chaos over the Internet of Things. Part of the phase diagram is shown in Figure 3.

Quantum genetic algorithm is an intelligent optimization algorithm that combines genetic algorithm and quantum computing. It has good fast convergence and global search capabilities. Compared with traditional genetic algorithm, quantum genetic algorithm has three obvious advantages. First, the quantum genetic algorithm uses qubits instead of single binary data to encode feasible solutions in the solution space. Secondly, the quantum genetic algorithm adds the operation of chromosome update through the quantum revolving door, instead of the simple crossover and mutation operations in the traditional genetic algorithm. Finally, each qubit is an indeterminate state, belonging to the superposition state of ${ }^{*} 0^{\prime}$ and $1^{\prime}$, so the decoding operation and the encoding operation are different, so the security of the encryption system can be increased.

In quantum genetic algorithm, the gene on the chromosome is not a certain value. Rather, it is expressed in terms of probability amplitude. Each qubit can represent "0" and ${ }^{*} 1^{\prime}$, or it can represent the superposition state of $0^{\prime}$ and "1". Therefore, compared with the traditional genetic algorithm, the qubit encoding method can contain more information under the same encoding length. And a qubit is represented as follows: 


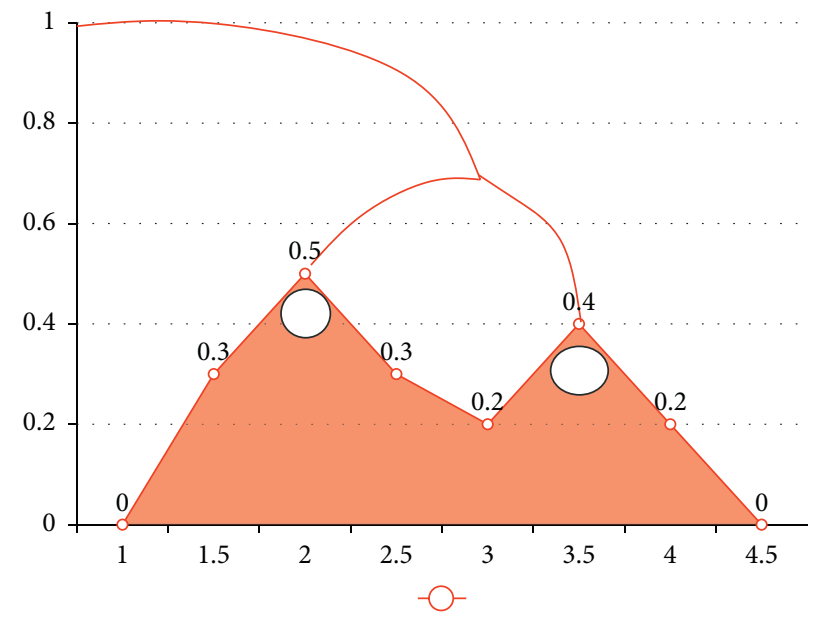

FIgURE 1: One-dimensional logistic map bifurcation diagram.

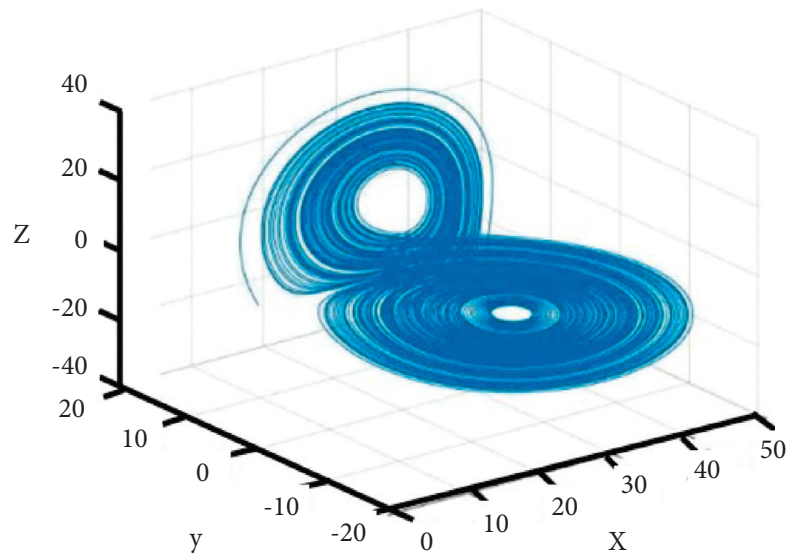

FIgURE 2: Lorenz IoT data chaotic system phase diagram: $x-y$ - $z$ three-dimensional view.

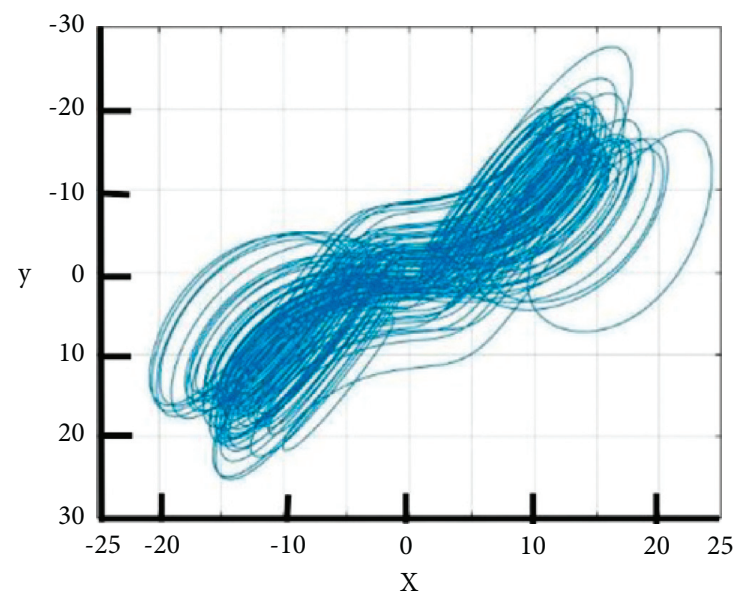

Figure 3: Chaos Lorenz system phase diagram of hyper-IoT data: $x-y$ two-dimensional projection. 


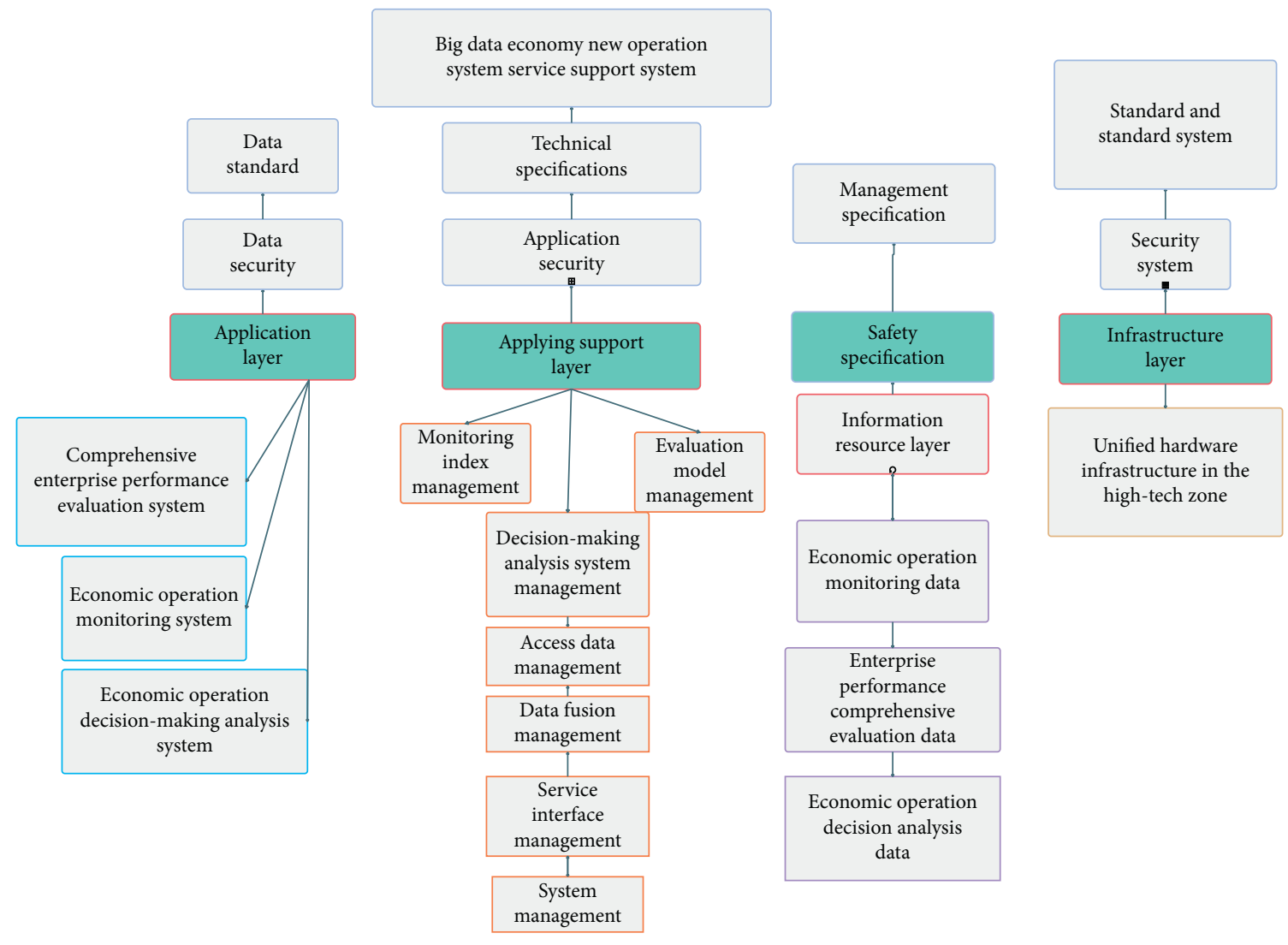

Overall architecture diagram of the new operating system of big data economy

FIgURE 4: The overall model of the system.

$$
\left[\begin{array}{l}
\alpha \\
\beta
\end{array}\right]
$$

Among them, $a$ and $\beta$ are complex numbers, representing the probability amplitudes of $0^{\prime}$ and " 1 ", respectively. And $a$ and $\beta$ satisfy the following formula:

$$
\alpha^{2}+\beta^{2}=1
$$

Therefore, the state of a qubit can be expressed as

$$
\psi=\alpha|0+\beta| 1 \text {. }
$$

In the quantum genetic algorithm, the chromosome is no longer a certain state under the abovementioned encoding method. The operations that generate the next generation cannot continue to use traditional selection, crossover, and mutation operations. However, the quantum spin gate acts on the state of quantum chromosomes, which makes them interfere with each other. In addition, the rotation of the quantum phase should be reversible, which ensures that the encryption process is reversible. Therefore, the distribution of the probability amplitude varies with the phase. The quantum revolving gate is described as follows:

$$
U=\left[\begin{array}{cc}
\cos \theta & -\sin \theta \\
\sin \theta & \cos \theta
\end{array}\right],
$$

and among them, $\theta$ is the phase value that needs to be rotated.

Therefore, the update process of each qubit is realized by formula (12).

$$
\left[\begin{array}{l}
\alpha^{\prime} \\
\beta^{\prime}
\end{array}\right]=U\left[\begin{array}{l}
\alpha \\
\beta
\end{array}\right] .
$$

The reverse process is described by the following equation:

$$
\left[\begin{array}{l}
\alpha^{\prime} \\
\beta^{\prime}
\end{array}\right]=U^{-1}\left[\begin{array}{l}
\alpha \\
\beta
\end{array}\right],
$$

and among them, $U^{-1}$ is the inverse matrix of $U$.

The particle swarm optimization algorithm is a speculative calculation method based on swarm intelligence. This similarity can be explained as follows: Suppose a group of birds randomly forage in a large field, and no bird will know the location of food in the field, but they only know the distance from the food. The best and effective way to find 


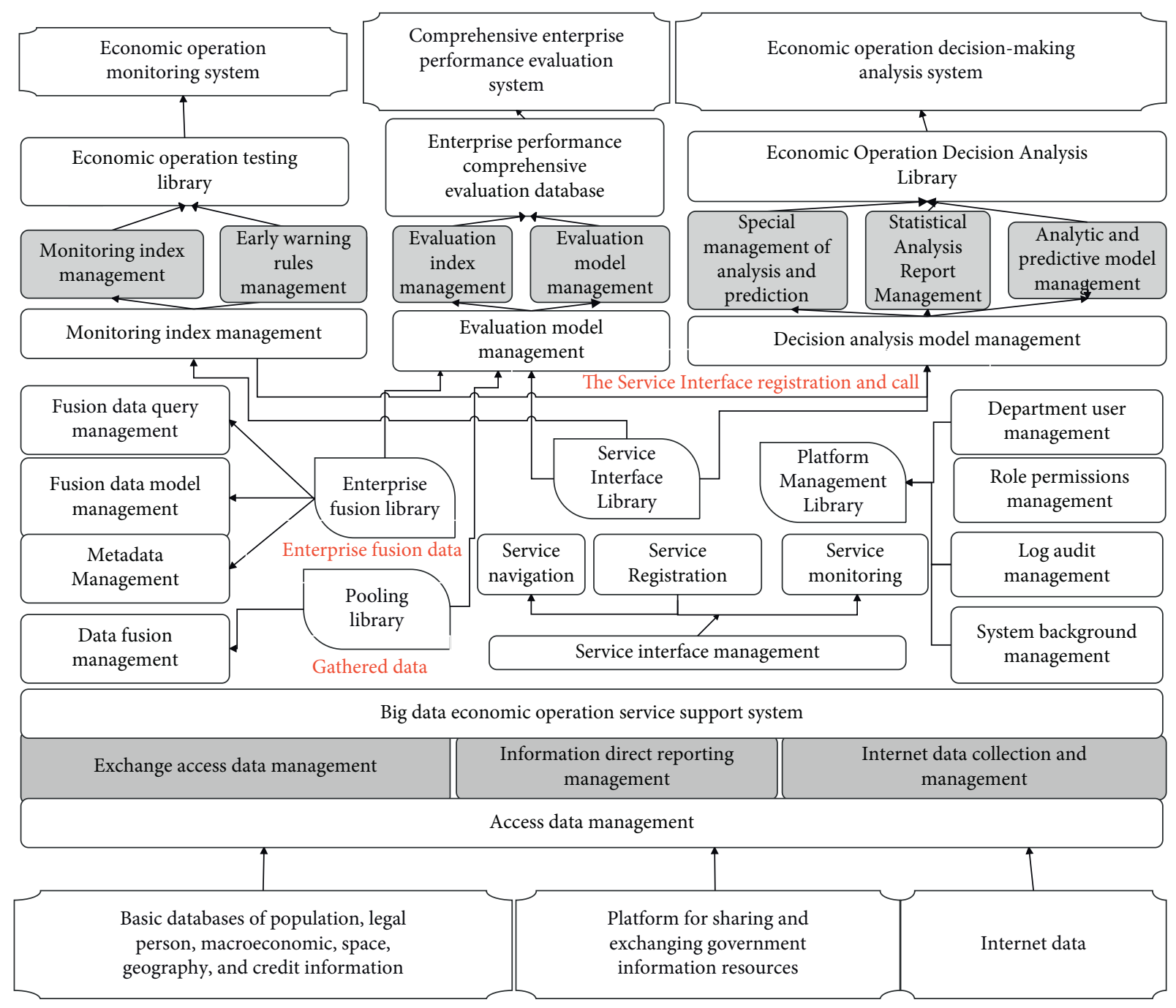

FIgURE 5: Architecture model of the service support system.

food is to be with the bird closest to the food location. The particle swarm optimization algorithm has the best ability to understand social behavior, so it is applied to find the solution space of various optimal problems.

Because the particle swarm optimization algorithm has the advantages of simple implementation and intuitive process, it has been widely used in many fields such as signal processing, machine learning, adaptive control, fuzzy system control, neural networks, function optimization, and model classification. Another advantage of the particle swarm optimization algorithm is that it only involves simple calculations and optimizes the problem by repeatedly trying to update the solution space related to a specific fitness function.

In the particle swarm optimization algorithm, each bird represents a separate solution, called a "particle." The algorithm starts from the initial state of any particle, and for each particle update, two optimal values are used: the first is the best value reached by the particle so far, which is called the particle best value (pbestt) and the second is the best value of the population which is called the best value gbest of all particles. All particles have three characteristics of fitness value, position, and speed, which guide the particles to find the best state of the individual and the best state of the population in the search space. The general algorithm of particle swarm optimization is described as follows.

The speed and position of each particle are updated according to the following formula:

$$
v_{k+1}=v_{k}+c l \times r l \times\left(\text { pbest }_{k}-p_{k}\right)+c 2 \times r 2 \times\left(\text { gbest }-p_{k}\right) .
$$

The particle swarm optimization algorithm generally has the following steps.

Step 1. Initialization process.

The algorithm sets constant values for $\mathrm{c} 1, \mathrm{c} 2, \mathrm{r} 1$, and 2, and the initial particle swarm is generated; The algorithm initializes the position and velocity of each particle randomly.

Step 2. Optimization process.

The algorithm calculates the fitness value of each particle: 


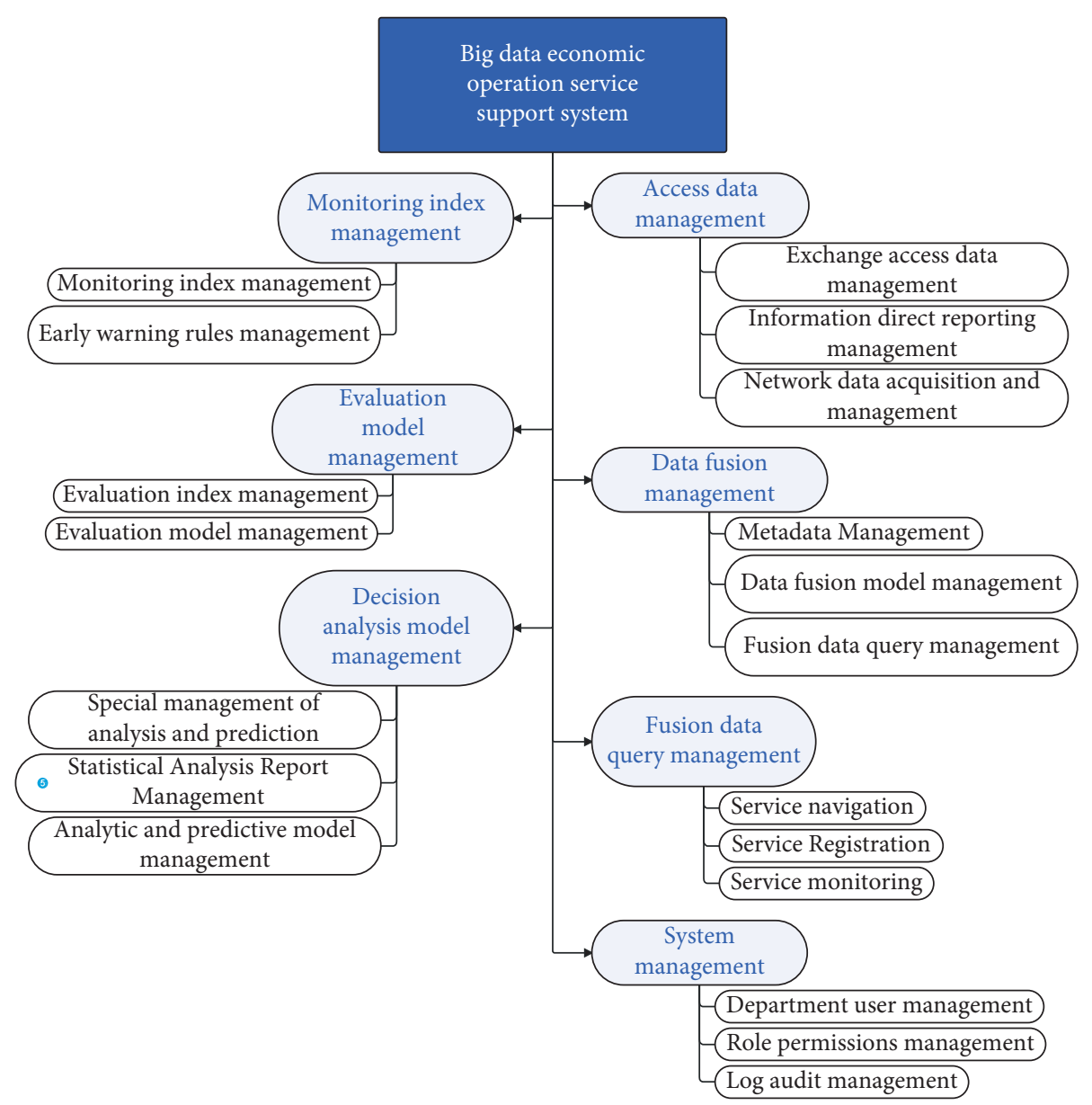

FIGURE 6: The functional structure of the service support system.

If the particle fitness value is better than the pbest of the previous particle, the algorithm updates pbest; otherwise it does not update; the best value of the updated particle swarm is gbest.

If (termination condition is met):

the algorithm proceeds to Step 3; else.

The algorithm updates the velocity of each particle through formula (14) and updates the position of each particle through formula (15), proceeding to Step 2.

Step 3. The algorithm terminates.

Compressed sensing technology can complete image encryption and compression operations in simple steps, where the measurement matrix is shared between the sender and the receiver as a key. In the process of digital image encryption, compressed sensing technology can effectively reduce data storage and improve the efficiency of encryption and decryption.

We set up a two-dimensional signal $x$ of size A and $\mathrm{K}$-sparse and describe it as

$$
x=\psi f,
$$

and among them, $f$ represents the transform coefficient vector. $\psi$ is an orthogonal basis of size $N \times N$. After that, the compressed signal $y$ is obtained by processing the measurement matrix of size $M \times N$. Then it can be expressed as

$$
y=\Phi x=\Phi \psi f=\eta f .
$$

In order to accurately reconstruct $x$ from $y$, the optimization problem can be solved by formula (17):

$$
\min \|f\|_{1} \text { s.t. } y=\eta f \text {. }
$$

The basic model of compressed sensing mainly includes three main steps: signal sparse representation, compressed measurement, and signal reconstruction. In image processing, sparse representation methods include Discrete Fourier Transform (DFT), Discrete Cosine Transform (DCT), Discrete Wavelet Transform (DWT), and so on. In terms of compression measurement, the measurement matrix generally includes Gaussian random matrix, partial orthogonal matrix, Hadamard matrix, and circulant matrix. This article uses a circulant matrix. In the signal reconstruction, the orthogonal matching pursuit (OMP) algorithm, the subspace pursuit (SP) algorithm, and the smooth L0 norm (SL0) algorithm are introduced. In this paper, the SL0 algorithm is used to restore the signal with the original size. 


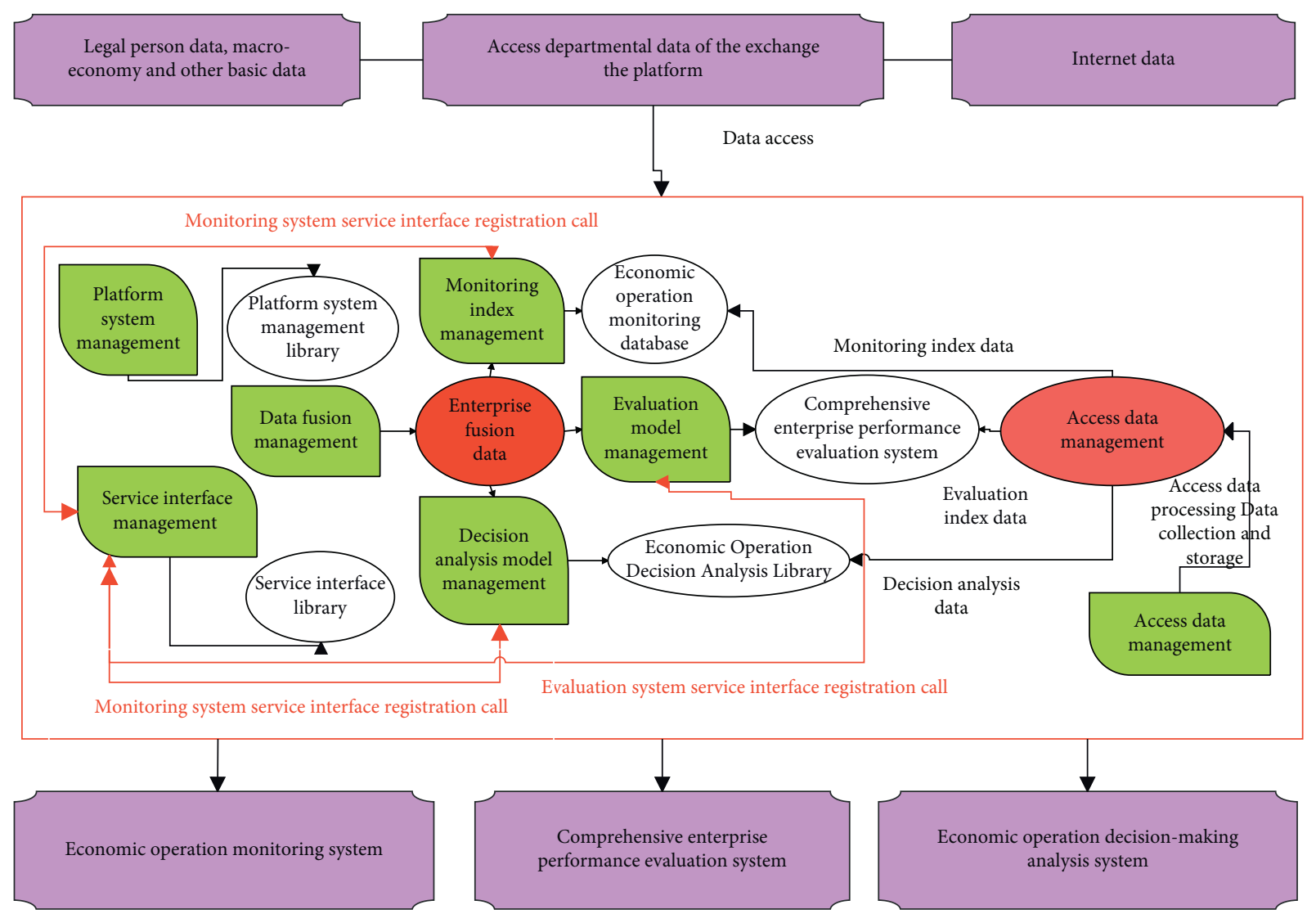

FIgURE 7: Data flow diagram of the service support system.

TABLE 1: Statistics on data acquisition and economic data mining capabilities of the Internet of Things.

\begin{tabular}{|c|c|c|c|c|c|c|c|c|}
\hline No. & IoT processing & Economic data mining & No. & IoT processing & Economic data mining & No. & IoT processing & Economic data mining \\
\hline 1 & 93.9 & 88.4 & 23 & 86.4 & 91.1 & 44 & 90.5 & 80.1 \\
\hline 2 & 96.9 & 82.6 & 24 & 95.1 & 87.3 & 45 & 86.8 & 85.4 \\
\hline 3 & 96.9 & 92.9 & 25 & 94.2 & 90.8 & 46 & 86.6 & 83.9 \\
\hline 4 & 86.2 & 87.2 & 26 & 85.6 & 87.4 & 47 & 86.0 & 84.4 \\
\hline 5 & 88.0 & 90.7 & 27 & 91.7 & 87.9 & 48 & 89.4 & 86.8 \\
\hline 6 & 88.2 & 80.4 & 28 & 92.5 & 80.4 & 49 & 93.5 & 89.8 \\
\hline 7 & 89.6 & 90.1 & 29 & 89.0 & 85.4 & 50 & 89.5 & 86.2 \\
\hline 8 & 88.0 & 80.2 & 30 & 96.7 & 85.0 & 51 & 91.4 & 85.4 \\
\hline 9 & 88.3 & 89.6 & 31 & 88.9 & 90.3 & 52 & 87.8 & 82.5 \\
\hline 10 & 87.9 & 82.4 & 32 & 89.8 & 89.5 & 53 & 88.6 & 88.3 \\
\hline 11 & 89.3 & 83.0 & 33 & 93.8 & 84.1 & 54 & 91.2 & 90.6 \\
\hline 12 & 93.0 & 88.8 & 34 & 95.8 & 87.2 & 55 & 89.3 & 80.1 \\
\hline 13 & 92.3 & 87.6 & 35 & 92.0 & 81.9 & 56 & 96.1 & 92.7 \\
\hline 14 & 89.8 & 84.4 & 36 & 96.2 & 90.8 & 57 & 85.7 & 89.5 \\
\hline 15 & 92.1 & 89.5 & 37 & 85.6 & 92.0 & 58 & 96.7 & 80.4 \\
\hline 16 & 96.4 & 82.9 & 38 & 91.5 & 90.8 & 59 & 86.1 & 92.6 \\
\hline 17 & 85.3 & 81.8 & 39 & 90.2 & 87.5 & 60 & 94.5 & 91.0 \\
\hline 18 & 88.6 & 87.6 & 40 & 87.7 & 85.9 & 61 & 87.1 & 84.8 \\
\hline 19 & 94.9 & 83.5 & 41 & 96.7 & 85.7 & 62 & 89.1 & 80.9 \\
\hline 20 & 85.1 & 92.1 & 42 & 95.1 & 85.1 & 63 & 94.4 & 86.0 \\
\hline 21 & 91.1 & 92.4 & 43 & 89.6 & 88.2 & 64 & 93.6 & 91.4 \\
\hline 22 & 87.6 & 86.8 & & & & & & \\
\hline
\end{tabular}


In this paper, the circulant matrix is applied as a measurement matrix, in which each row is generated by moving one bit to the right from the previous row. In addition, the first row vector is generated by the hyper-IoT data chaotic system. The measurement matrix $\Phi$ with a size of $M \times N$ can be described as

$$
\left\{\begin{array}{l}
\Phi(i, 1)=\mu \times \Phi(i-1, N) \\
\Phi(i, 2: N)=\Phi(i-1,1: N-1)
\end{array}\right.
$$

and among them, $2 \leq i \leq M$ and $\mu>1$. This paper adopts a hyper-IoT data chaos system that is highly sensitive to initial values and system parameter values. Therefore, when the initial values and system parameter values change slightly, we can get completely different measurement matrices, which will produce completely different compression effects.

\section{Analysis System of Economic Development Trend Based on Big Data in the Internet of Things Environment}

The economic operation system model is divided into infrastructure layer, information resource layer, application support layer, application layer, standard specification system, and safety guarantee system. The information resource layer is composed of data accessed and managed by the platform, and the application support layer is composed of an economic operation service support system. The application layer is composed of the economic operation monitoring system, the enterprise performance comprehensive evaluation system, the economic operation decision analysis system, and the high-tech zone enterprise service system. The standard specification system is composed of data standards, technical specifications, and management specifications, and the security assurance system is composed of data security, application security, and security management. The overall model design is shown in Figure 4.

The system architecture of the economic operation service support system based on big data is shown in Figure 5.

The system includes functions such as access data management, data fusion management, service interface management, platform system management, economic operation monitoring index management, enterprise performance comprehensive evaluation model management, and economic operation decision analysis model management. The system function structure of the economic operation service support system based on big data is shown in Figure 6.

Data collection and access, storage, processing integration, and processing to classification services constitute a complete data flow of the system. Data sources include basic data such as legal persons and macroeconomics, exchange platform access data, and Internet data. After the data is accessed, it is stored in the collection library, which can be directly called by the subsystem database, or it can be called after processing and fusion. The specific system data flow is shown in Figure 7.

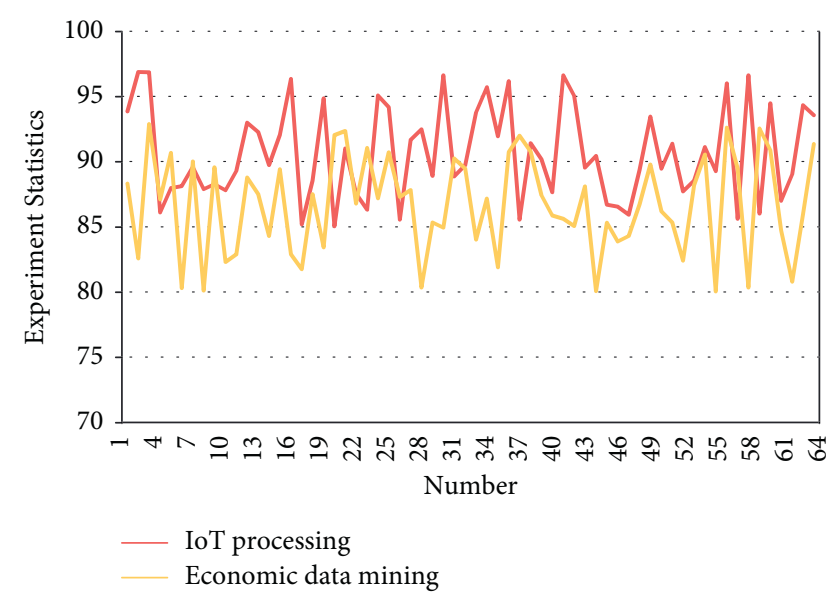

Figure 8: Results of experiment statistics.

After constructing the above model, the performance of the model is verified. This paper takes the enterprise data of a high-tech zone as an example to carry out research and evaluation on the effect of this system and calculates the ability of the Internet of Things to obtain data economy and explores the system's economic data mining ability. Through these data, the performance of the system proposed in this paper is verified, and the data acquisition and economic data mining capabilities of the Internet of Things are counted. The results obtained are drawn into charts as shown in Table 1 and Figure 8.

Through experimental research, it can be known that the system model proposed in this paper has a good performance in the forecast of economic development trend, and the system can be used for forecasting in the follow-up economic development forecast.

\section{Conclusion}

The rapid development of information technology has put a large number of application systems and new technologies into the analysis of economic development. Based on the Internet of Things technology, this paper designs an economic operation system model based on big data and develops a set of practical application systems. The system can not only solve the obstacles of data transmission, sharing, storage, etc., caused by the increasingly prominent phenomenon of information islands, but also better monitor the economic operation status. At the same time, it can provide support for relevant departments to grasp the latest economic development trend in a timely manner and provide objective factual basis for scientific decision-making. In addition, this paper constructs the system function structure based on the actual situation and analyzes the operation process of the system. The experimental research results show that the system model proposed in this paper has a good performance in the forecast of economic development trends, and the system can be used for forecasting in subsequent economic development forecasts. 


\section{Data Availability}

The labeled dataset used to support the findings of this study are available from the corresponding author upon request.

\section{Conflicts of Interest}

The author declares no conflicts of interest.

\section{Acknowledgments}

This study was sponsored by Xinyang Vocational and Technical College

\section{References}

[1] V. Daksiya, H. T. Su, Y. H. Chang, and E. Y. M. Lo, "Incorporating socio-economic effects and uncertain rainfall in flood mitigation decision using MCDA," Natural Hazards, vol. 87, no. 1, pp. 515-531, 2017.

[2] S. Lahmiri, "A variational mode decompoisition approach for analysis and forecasting of economic and financial time series," Expert Systems with Applications, vol. 55, no. 8, pp. 268-273, 2016.

[3] N. Gordini, "A genetic algorithm approach for SMEs bankruptcy prediction: empirical evidence from Italy," Expert Systems with Applications, vol. 41, no. 14, pp. 6433-6445, 2014.

[4] A. Ferramosca, A. H. González, D. Limon, and D. Limon, "Offset-free multi-model economic model predictive control for changing economic criterion," Journal of Process Control, vol. 54 , no. 3 , pp. 1-13, 2017.

[5] C. J. A. Jane, "Hybrid model combined grey prediction and autoregressive integrated moving average model for talent prediction," Journal of Grey System, vol. 21, no. 2, pp. 91-102, 2018.

[6] A. Khadjeh Nassirtoussi, S. Aghabozorgi, T. Ying Wah, and D. C. L. Ngo, "Text mining for market prediction: a systematic review," Expert Systems with Applications, vol. 41, no. 16, pp. 7653-7670, 2014.

[7] M. Ellis and P. D. Christofides, "Integrating dynamic economic optimization and model predictive control for optimal operation of nonlinear process systems," Control Engineering Practice, vol. 22, no. 1, pp. 242-251, 2014.

[8] W. Schultz, W. R. Stauffer, and A. Lak, "The phasic dopamine signal maturing: from reward via behavioural activation to formal economic utility," Current Opinion in Neurobiology, vol. 43 , no. 5, pp. 139-148, 2017.

[9] O. Sosnovska and M. Zhytar, "Financial architecture as the base of the financial safety of the enterprise," Baltic Journal of Economic Studies, vol. 4, no. 4, pp. 334-340, 2018.

[10] D. Acemoglu, A. Ozdaglar, and A. Tahbaz-Salehi, "Systemic risk and stability in financial networks," The American Economic Review, vol. 105, no. 2, pp. 564-608, 2015.

[11] R. Castellano, R. Cerqueti, and G. Rotundo, "Exploring the financial risk of a temperature index: a fractional integrated approach," Annals of Operations Research, vol. 284, no. 1, pp. 225-242, 2020.

[12] A. Cohn, J. Engelmann, E. Fehr, and M. A. Maréchal, "Evidence for countercyclical risk aversion: an experiment with financial professionals," The American Economic Review, vol. 105, no. 2, pp. 860-885, 2015.

[13] M. Maggiori, "Financial intermediation, international risk sharing, and reserve currencies," The American Economic Review, vol. 107, no. 10, pp. 3038-3071, 2017.
[14] I. Pinelis, "An optimal three-way stable and monotonic spectrum of bounds on quantiles: a spectrum of coherent measures of financial risk and economic inequality," Risks, vol. 2, no. 3, pp. 349-392, 2014.

[15] N. Hautsch, J. Schaumburg, and M. Schienle, "Financial network systemic risk contributions," Review of Finance, vol. 19, no. 2, pp. 685-738, 2015.

[16] C. N. Noussair, S. T. Trautmann, and G. Van de Kuilen, "Higher order risk attitudes, demographics, and financial decisions," The Review of Economic Studies, vol. 81, no. 1, pp. 325-355, 2014.

[17] B. Heidenreich, M. Reece, and T. Rudelius, "Weak gravity strongly constrains large-field axion inflation," Journal of High Energy Physics, vol. 2015, no. 12, pp. 1-41, 2015.

[18] O. Coibion, Y. Gorodnichenko, and R. Kamdar, "the formation of expectations, inflation, and the phillips curve," Journal of Economic Literature, vol. 56, no. 4, pp. 1447-1491, 2018.

[19] J. L. Yellen, "Inflation, uncertainty, and monetary policy," Business Economics, vol. 52, no. 4, pp. 194-207, 2017.

[20] M. McLeay and S. Tenreyro, "Optimal inflation and the identification of the phillips curve," NBER Macroeconomics Annual, vol. 34, no. 1, pp. 199-255, 2020.

[21] J. Vavra, "Inflation dynamics and time-varying volatility: new evidence and an ss interpretation," The Quarterly Journal of Economics, vol. 129, no. 1, pp. 215-258, 2014.

[22] A. Cavallo, G. Cruces, and R. Perez-Truglia, "Inflation expectations, learning, and supermarket prices: evidence from survey experiments," American Economic Journal: Macroeconomics, vol. 9, no. 3, pp. 1-35, 2017. 